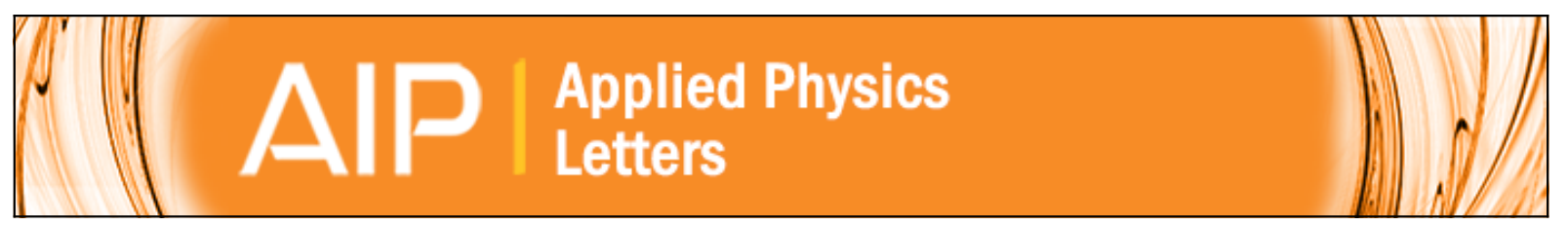

\title{
Surface effects of electrode-dependent switching behavior of resistive random-access memory
}

Jr-Jian Ke, Tzu-Chiao Wei, Dung-Sheng Tsai, Chun-Ho Lin, and Jr-Hau He

Citation: Applied Physics Letters 109, 131603 (2016); doi: 10.1063/1.4963671

View online: http://dx.doi.org/10.1063/1.4963671

View Table of Contents: http://scitation.aip.org/content/aip/journal/apl/109/13?ver=pdfcov

Published by the AIP Publishing

\section{Articles you may be interested in}

Multi-step resistive switching behavior of Li-doped $\mathrm{ZnO}$ resistance random access memory device controlled by compliance current

J. Appl. Phys. 119, 244506 (2016); 10.1063/1.4955063

Unipolar resistive switching behavior of $\mathrm{Pt} / \mathrm{Li}$ x Zn1- $\mathrm{Z}$ O/Pt resistive random access memory devices controlled by various defect types

Appl. Phys. Lett. 101, 203501 (2012); 10.1063/1.4766725

Insight into distribution and switching of resistive random-access memory filaments based on analysis of variations in memory characteristics

J. Appl. Phys. 112, 044503 (2012); 10.1063/1.4745056

Surface effect on resistive switching behaviors of $\mathrm{ZnO}$

Appl. Phys. Lett. 99, 192106 (2011); 10.1063/1.3659296

Characteristics and mechanism of conduction/set process in $\mathrm{Ti} N / \mathrm{Zn} \mathrm{O} / \mathrm{Pt}$ resistance switching random-access memories

Appl. Phys. Lett. 92, 232112 (2008); 10.1063/1.2945278

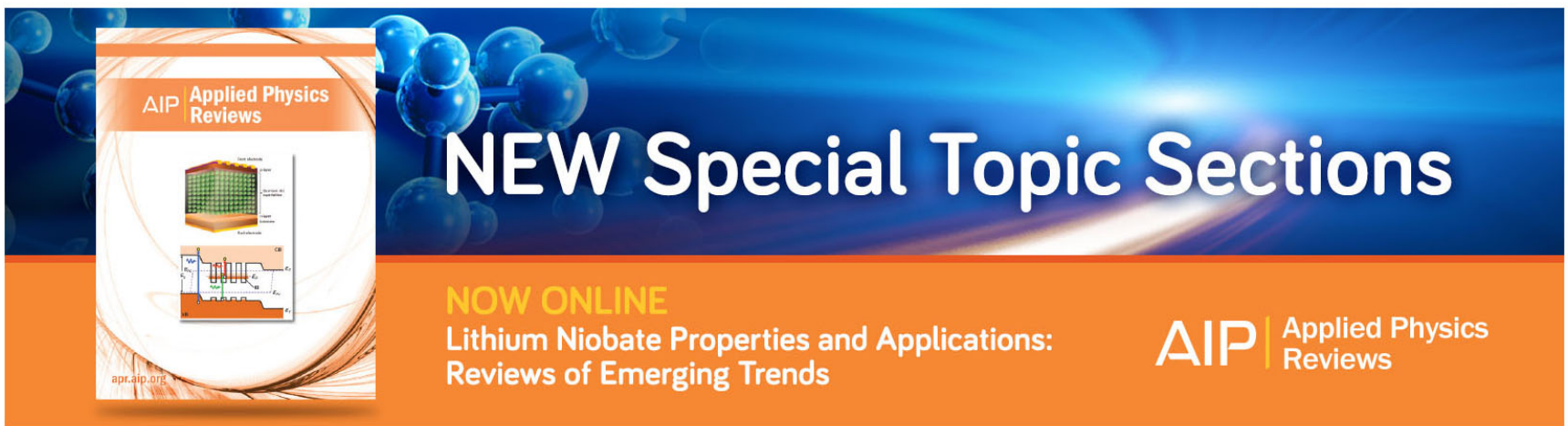




\title{
Surface effects of electrode-dependent switching behavior of resistive random-access memory
}

\author{
Jr-Jian Ke, ${ }^{1,2}$ Tzu-Chiao Wei, ${ }^{2}$ Dung-Sheng Tsai, ${ }^{2}$ Chun-Ho Lin, ${ }^{1}$ and Jr-Hau He ${ }^{1, a)}$ \\ ${ }^{1}$ Computer, Electrical and Mathematical Sciences and Engineering (CEMSE) Division, \\ King Abdullah University of Science and Technology (KAUST), Thuwal 23955-6900, Saudi Arabia \\ ${ }^{2}$ Institute of Photonics and Optoelectronics and Department of Electrical Engineering, \\ National Taiwan University, Taipei, Taiwan
}

(Received 11 June 2016; accepted 14 September 2016; published online 27 September 2016)

\begin{abstract}
The surface effects of ZnO-based resistive random-access memory (ReRAM) were investigated using various electrodes. Pt electrodes were found to have better performance in terms of the device's switching functionality. A thermodynamic model of the oxygen chemisorption process was proposed to explain this electrode-dependent switching behavior. The temperature-dependent switching voltage demonstrates that the ReRAM devices fabricated with Pt electrodes have a lower activation energy for the chemisorption process, resulting in a better resistive switching performance. These findings provide an in-depth understanding of electrode-dependent switching behaviors and can serve as design guidelines for future ReRAM devices. Published by AIP Publishing.

[http://dx.doi.org/10.1063/1.4963671]
\end{abstract}

The surface effects of metal oxides, including chemisorption, photodesorption, and surface roughness, result in surface band bending which can significantly influence the electronic and optoelectronic properties of the material. For example, the surface band bending of $\mathrm{ZnO}$ due to chemisorbed oxygen molecules is around $1.53 \mathrm{eV}$. ${ }^{1}$ The lower conductivity as a result of this surface band bending makes $\mathrm{ZnO}$ a promising candidate for photodetector and gas sensing applications.

Recently, the development of resistive random-access memory (ReRAM) has attracted a great deal of attention due to its superior characteristics of high-speed operation, lowpower consumption, and long retention time. ${ }^{2-4}$ Among the metal oxides currently being explored to fabricate ReRAM, $\mathrm{ZnO}$ has been demonstrated as a potential candidate. $\mathrm{ZnO}$ is transparent in the visible spectral region and its electrical properties can be modulated using suitable impurity doping; therefore, it is a promising material for the fully transparent ReRAM which could be used in transparent electronics and displays. ${ }^{5}$

The switching mechanism of $\mathrm{ZnO}$ ReRAM is mainly attributed to the electrochemical redox process associated with the formation/rupture of conductive nanofilaments built by oxygen vacancies $\left(\mathrm{V}_{O}\right)$ near the electrode/ $\mathrm{ZnO}$ interface. ${ }^{6}$ The switching functionality is enormously influenced by chemisorbed oxygen adatoms $\left(\mathrm{O}_{2(a d)}^{-}\right)$at the surface. ${ }^{7-9}$ For instance, Yang et al. reported the ambient effects on transparent ReRAM can be remarkably suppressed by introducing graphene electrodes as a surface passivation layer. ${ }^{7}$

Many reports have shown that the resistive switching properties of ReRAM significantly depend on the top electrode. These studies have mainly focused on the transport mechanisms of ReRAM (i.e., space charge limited current, or Poole-Frenkel emission), switching behavior (unipolar or bipolar), and the filament type (e.g., oxygen vacancies or

${ }^{a)}$ E-mail: jrhau.he@kaust.edu.sa cation migration-induced metallization). ${ }^{10-12}$ The interfacial reaction between the metal electrode and the active layer may cause either a favorable or an adverse influence on the properties of the ReRAM's resistive switching behavior. ${ }^{13,14}$ Researchers have previously reported the resistive switching properties of $\mathrm{ZnO}$ with various metal electrodes. ${ }^{11,15}$ However, there has not yet been a systematic investigation of how surface effects influence the metal electrode dependence of $\mathrm{ZnO}$ ReRAM.

In this work, we investigated how surface effects may change the electrical properties of $\mathrm{ZnO}$ ReRAM devices fabricated with various top electrodes (e.g., Pt, Au, and $\mathrm{Cr}$ ). In our results, the surface effects of $\mathrm{ZnO}$ were investigated by the thickness-dependent electrical behavior initially. In addition, we examined how different electrical characteristics of $\mathrm{ZnO}$ ReRAM, including the switching probability, high-resistance state (HRS), and low-resistance state (LRS), changed with various top electrodes and ambient conditions. From this work, we learned that $\mathrm{ZnO}$ ReRAM made with Pt electrodes possesses a higher resistive switching probability, showing a better electrical performance. Analysis of the temperature-dependent SET/ RESET voltage for each top electrode metal showed that the Pt electrode also had the lowest activation energy for the chemisorption of oxygen molecules. This indicates that the chemisorption processes are more pronounced, which increases the probability in the generation/elimination of oxygen vacancies near the $\mathrm{Pt} / \mathrm{ZnO}$ interface, improving the switching functionality of the $\mathrm{ZnO}$ ReRAM device. These observations advance our understanding of surface effects and provide valuable insights for future optimization of ReRAM devices.

$\mathrm{ZnO}$ thin films with thicknesses of $70,100,130$, and $160 \mathrm{~nm}$ were deposited on the $\mathrm{Pt} / \mathrm{Cr} / \mathrm{SiO}_{2} / \mathrm{Si}$ substrates at room temperature by RF-magnetron sputtering, using an Ar working pressure of $5 \mathrm{mTorr}$ and a ceramic $\mathrm{ZnO}$ target. In order to study the electrode dependence of ZnO ReRAM, the 
circular-shaped top electrodes of $\mathrm{Pt}, \mathrm{Au}$, and $\mathrm{Cr}(100 \mathrm{~nm}$ thick and $200 \mu \mathrm{m}$ in diameter) were deposited on top of the $\mathrm{ZnO}$ thin films. The top electrodes were patterned using a shadow mask. Note: the $\mathrm{Cr}$ electrode was capped by $\mathrm{Pt}$ $(50 \mathrm{~nm}$ thick) to prevent oxidation.

The $\mathrm{ZnO}$ ReRAM devices with these different top electrodes were measured under air and vacuum conditions using a Keithley 4200 parameter analyzer to study how surface effects influenced the electrode dependence of $\mathrm{ZnO}$ ReRAM. To gain further insight into the electrical characteristics, the devices were measured at temperatures ranging from $100-350 \mathrm{~K}$ to study the temperature dependence of the SET/RESET switching voltage characteristics. Fig. 1(a) shows the schematic of the ReRAM device with Pt, Au, and $\mathrm{Cr}$ as the top electrode, as well as the corresponding room temperature I-V curves, which were taken in air.

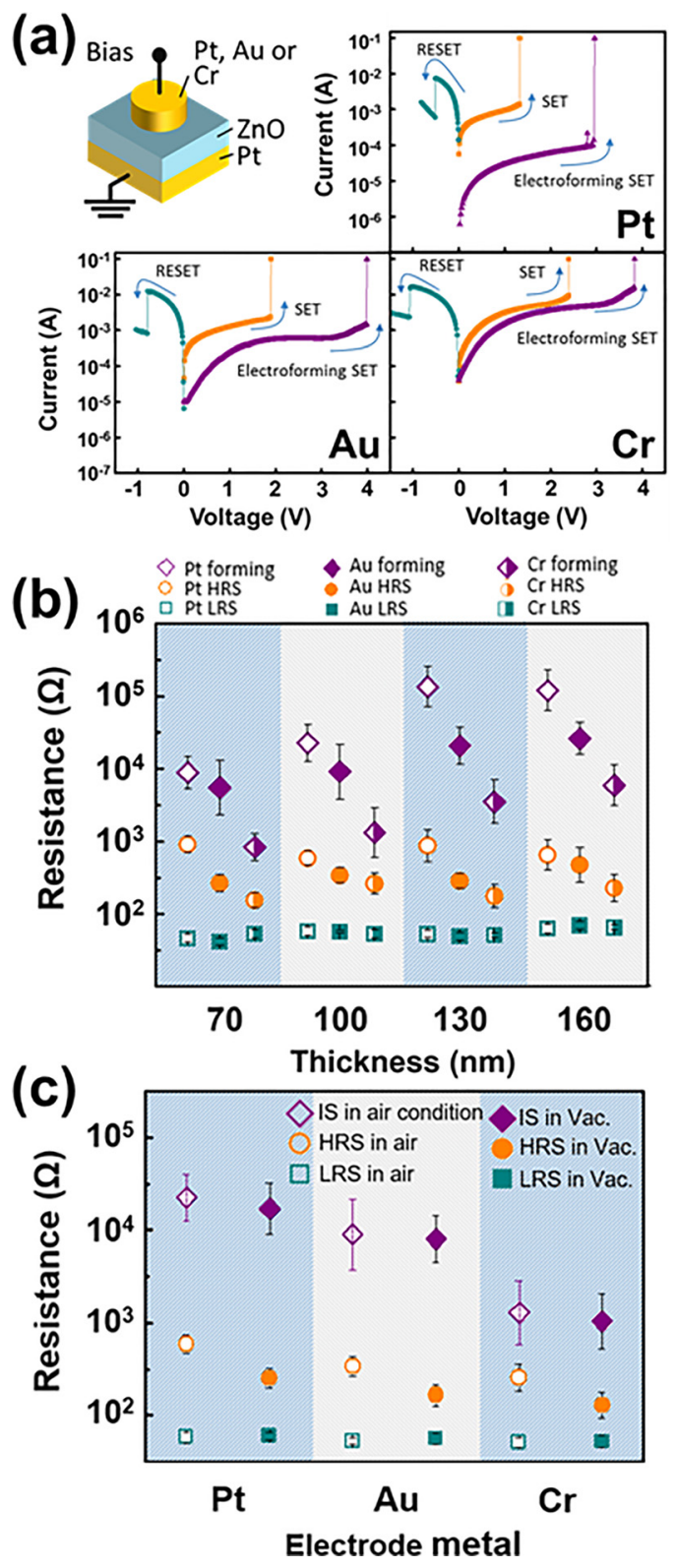

FIG. 1. (a) A schematic of the ZnO ReRAM device and the corresponding I-V curves for $\mathrm{Pt}, \mathrm{Au}$, and $\mathrm{Cr}$ as the top-electrode. (b) IS, HRS, and LRS resistance with various $\mathrm{ZnO}$ film thicknesses, and (c) under air and vacuum conditions for $\mathrm{Pt}, \mathrm{Au}$, and $\mathrm{Cr}$ top electrodes.
Fig. 1(b) shows how the resistance of the initial state (IS), HRS, and LRS changed for the ReRAM devices with different $\mathrm{ZnO}$ film thicknesses and under various top electrode conditions. The results demonstrate that for each top electrode metal, the IS resistance was thickness-dependent, while the HRS and LRS were thickness-independent. Note that we obtained the statistics for each dataset using 30 different ReRAM devices.

The ReRAM switching function relies on the formation/ rupture of conductive nanofilaments built by oxygen vacancies, $\mathrm{V}_{O}$. As the $\mathrm{ZnO}$ ReRAM devices were electroformed, conductive nanofilaments dominated the electrical transport within the bulk $\mathrm{ZnO}$, causing the HRS and LRS resistance to be independent of the $\mathrm{ZnO}$ film thickness. Because the switching process does not change as a result of the $\mathrm{ZnO}$ film thickness, it suggests that changes to the device resistance occur at the surface, or more specifically, near the electrode $/ \mathrm{ZnO}$ interface.

Fig. 1(b) also shows the IS and HRS resistances are electrode-dependent for films of the same thickness, decreasing in the order of $\mathrm{Pt}>\mathrm{Au}>\mathrm{Cr}$. The electrode dependence can be attributed to two mechanisms occurring near the electrode/ZnO interface. First, this electrode-dependent resistance could originate from contact resistance due to $\mathrm{ZnO}$ band bending near the metal contact region, which is determined by the work function of the metal electrode. The work function of $\mathrm{Pt}, \mathrm{Au}$, and $\mathrm{Cr}$ is $5.93,5.31$, and $4.50 \mathrm{eV}$, respectively. ${ }^{16} \mathrm{~A}$ high work function results in high contact resistance of the electrode to the metal oxide, leading to the electrode dependence shown in Fig. 1(b). The second mechanism that may cause the device's electrode dependent resistance is chemisorption from the ambient environment, which tends to capture free electrons at the surface and form a lowconductivity depletion layer near the surface of the metal oxide to reduce the IS and HRS resistance of the ReRAM. ${ }^{17}$

In order to determine the origin of the electrode dependence, we measured the resistance of the $\mathrm{ZnO}$ ReRAM device under different ambient environments. Fig. 1(c) shows the IS, HRS, and LRS resistances measured under air and vacuum conditions. Clearly, the HRS is influenced by the environment, while the ambient effects on IS and LRS are not significant. These results indicate that chemisorption plays an important role in determining the device's HRS. This phenomenon can be understood by the lower conductivity near the $\mathrm{ZnO}$ surface that is induced by oxygen chemisorption. ${ }^{18}$

The surface band bending effect is more pronounced as the $\mathrm{O}_{2(a d)}^{-}$concentration increases, resulting in the electrode dependence of the device's HRS. On the other hand, the IS is not electrode dependent, suggesting that the bulk component overwhelms surface effects, since there are no conductive paths within the bulk of the device prior to the electroforming process. No obvious change in the LRS confirms that transport is dominated by the conductive nanofilaments and that surface band bending has negligible effect.

To further investigate the surface effects of $\mathrm{ZnO}$ ReRAM, we also investigated the electrode-dependent switching functionality of the devices under various ambient conditions. We defined the switching probability as the ratio of the number of devices switching continually over 3 cycles 
of the SET/RESET process without any failure to the number of total devices tested (30 cells). As shown in Fig. 2, the switching probability was highest for the ReRAM devices made with Pt electrodes, followed by $\mathrm{Au}$ and $\mathrm{Cr}$. The concentration of chemisorbed $\mathrm{O}_{2(a d)}^{-}$at the surface of metal oxides at room temperature is greater in air compared to vacuum. ${ }^{17,18}$ Our results in Fig. 2 show that the devices tested under air had a higher switching probability compared to the same ReRAM devices tested under vacuum, which suggests that a higher $\mathrm{O}_{2(a d)}^{-}$concentration leads to a higher switching probability. These findings are in line with Ke et al., who reported that in order to achieve a high switching probability, a high concentration of chemisorbed $\mathrm{O}_{2(a d)}^{-}$can be created by either changing the ambient environment or the surface roughness of the metal oxide, which enables $\mathrm{O}_{2(a d)}^{-}$to help generate/eliminate $\mathrm{V}_{O}$ near the electrode/ZnO interface. ${ }^{19}$ The pronounced chemisorption process on the surface results in the higher formation/rupture probability of $\mathrm{V}_{O}$-built nanofilaments, leading to the better switching functionality. In this study, the high switching probability shown in $\mathrm{ZnO}$ ReRAM fabricated with Pt electrodes suggests that oxygen chemisorption near the $\mathrm{Pt}$ is more pronounced compared to other metal electrodes.

It is widely known that the $\mathrm{ZnO}$ surface is very sensitive to the ambient environment due to oxygen chemisorption. ${ }^{17,18}$ The ambient sensitivity of $\mathrm{ZnO}$ can be further increased using Pt or Au nanoparticles; a finding which is attributed to the fact that $\mathrm{Pt}$ and $\mathrm{Au}$ serve as catalysts to lower the activation energy for both the desorption/adsorption processes. ${ }^{20-22}$ In addition, gas sensors made using Pt/ $\mathrm{ZnO}$ stacks, with capping layers on the edges of the Pt top electrodes, show that the ambient environment can directly affect the chemisorption process at the $\mathrm{ZnO}$ surface through the Pt layer due to the high mobility of oxygen within $\mathrm{Pt}^{23,24}$ This indicates that the chemisorption of $\mathrm{O}_{2(a d)}^{-}$near the Pt electrode is more pronounced.

For the ReRAM device, the chemisorption process, involving the desorption/adsorption of $\mathrm{O}_{2(a d)}^{-}$, increases the probability of the generation/elimination of oxygen vacancies. The desorption of $\mathrm{O}_{2(a d)}^{-}$leaves a vacant adatom site, which increases the probability that oxygen vacancies will build conductive nanofilaments and induce the SET process near the electrode/ $\mathrm{ZnO}$ interface. The temperature and

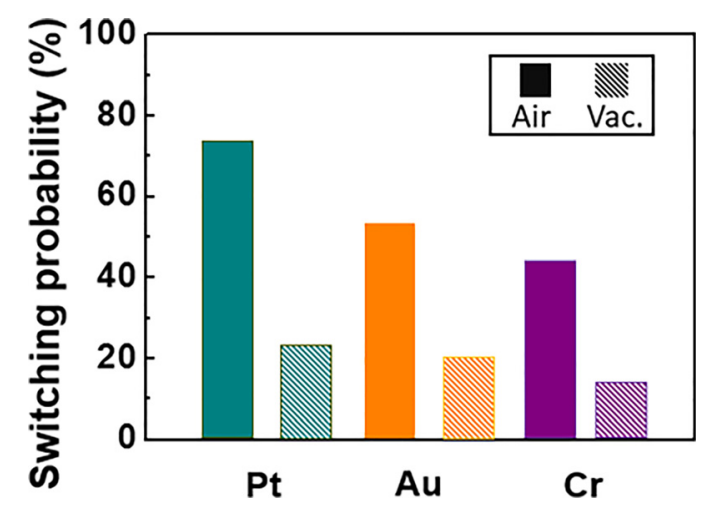

FIG. 2. The switching probability for $\mathrm{ZnO}$ ReRAM with $\mathrm{Pt}, \mathrm{Au}$, and $\mathrm{Cr}$ top electrodes. applied bias significantly affect the desorption/adsorption process at the $\mathrm{ZnO}$ surface. High temperature leads to a pronounced desorption/adsorption process, due to thermal activation. ${ }^{1}$ In addition, an enhanced desorption/adsorption process was also observed with the applied bias. The underlying mechanism was attributed to the field-induced rearrangement of the free electron distribution, which tends to affect the chemisorption process. ${ }^{25-27}$

To further study the electrode dependence on the electrical properties, we investigated the temperature-dependent resistive switching behaviors of $\mathrm{ZnO}$ ReRAM. As shown in Fig. 3(a), both the SET/RESET switching voltages decreased in the order of $\mathrm{Cr}>\mathrm{Au}>\mathrm{Pt}$, and they decreased with rising temperature. To gain insight into the phenomenon observed in Figs. 3(a) and 2, we assumed for the device's SET process that the chemisorption-assisted generation of $\mathrm{V}_{O}$ for building conductive nanofilaments happens at a threshold concentration of vacant adatom sites, $N_{O}$, while applying the SET voltage, $V_{S E T}$. The relationship between $N_{O}$ and $V_{S E T}$ can be written as

$$
N_{O} \propto \exp \left[-\left(E_{a \_S E T}-\alpha V_{S E T}\right) / k_{B} T\right],
$$

where $k_{B}$ is the Boltzmann constant, $T$ is the temperature, $\alpha V_{S E T}$ is the portion of the applied voltage $\left(V_{S E T}\right)$ near the electrode/ZnO interface to SET the resistive states of the ReRAM, and the $E_{a_{-} S E T}$ is the activation energy of $\mathrm{O}_{2(a d)}^{-}$ desorption. ${ }^{28,29}$

On the other hand, the RESET process relies on the rupture of the nanofilaments by recovery of the $\mathrm{V}_{O}$ sites near the electrode/ $\mathrm{ZnO}$ interface. The $\mathrm{O}_{2(a d)}^{-}$adsorbed on the $\mathrm{ZnO}$ surface tends to migrate and recombine with $\mathrm{V}_{O}$ to break these conductive nanofilaments, which helps the RESET process. Assuming the RESET process is triggered when the $\mathrm{O}_{2(a d)}^{-}$concentration on the $\mathrm{ZnO}$ surface reaches a critical value, $N_{a d}$, while applying the RESET voltage, $V_{R E S E T}$, then $N_{a d}$ can be written as a function of $V_{R E S E T}$ as

$$
N_{a d} \propto \exp \left[-\left(E_{a \_R E S E T}-\alpha V_{R E S E T}\right) / k_{B} T\right],
$$

where $E_{a \_R E S E T}$ is the activation energy of the RESET process associated with oxygen adsorption, and $\alpha V_{R E S E T}$ is the portion of the applied voltage $\left(V_{R E S E T}\right)$ near the electrode/ $\mathrm{ZnO}$ interface to RESET the ReRAM. ${ }^{28,29}$ By rearranging the terms in Eqs. (1) and (2), we obtain the relations shown in Eqs. (3) and (4). For the SET process, the equation is as follows:

$$
V_{S E T}=\frac{E_{a S E T}}{\alpha}+\left[\operatorname{Ln}\left[\frac{N_{O}}{A}\right] \alpha^{-1} k_{B}\right] T,
$$

where $A$ is a constant. For the RESET process, the equation is as follows:

$$
V_{R E S E T}=\frac{E_{a \_R E S E T}}{\alpha}+\left[\operatorname{Ln}\left[\frac{N_{a d}}{B}\right] \alpha^{-1} k_{B}\right] T,
$$

where $B$ is a constant. Using Eqs. (3) and (4), the activation energy of the desorption/adsorption of oxygen adatoms for the SET/RESET process can be estimated by the y-intercept of the $V_{\text {SET }} / V_{\text {RESET }}$ plotted against $T$. Fig. 3 shows the 

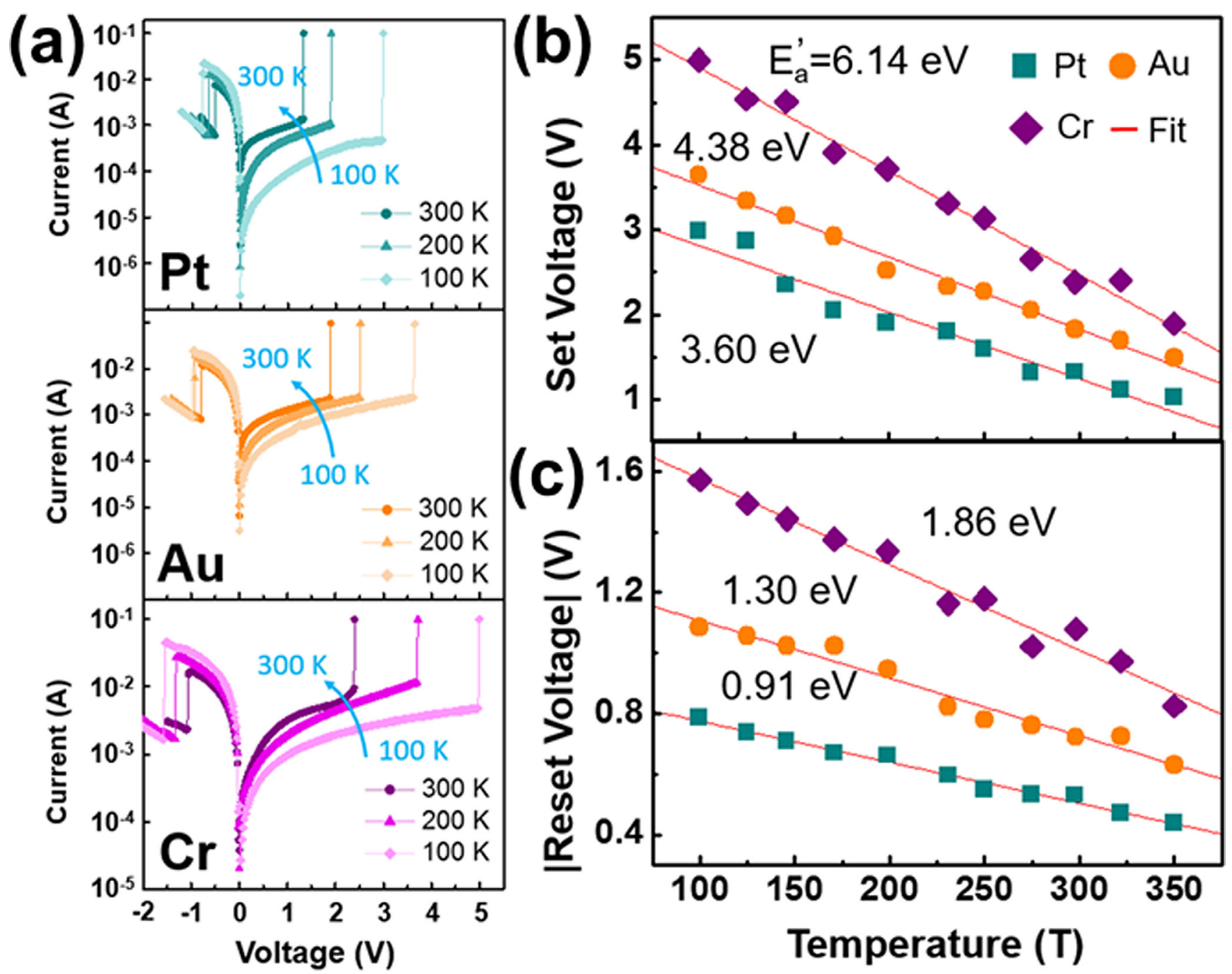

FIG. 3. (a) Temperature-dependent I-V curves for Pt, Au, and Cr top-electrode conditions. (b) The temperature-dependent SET voltage and (c) RESET voltage with line fittings for each electrode metal measured in the air. $E_{a}^{\prime}$ in (b) and (c) represents $E_{a_{-} S E T} / \alpha$ and $E_{a \_} R E S E T / \alpha$ in the text, respectively.

temperature-dependent SET/RESET voltage and its line fit, in which the y-intercept of Figs. 3(b) and 3(c) represent the $E_{a \_S E T} / \alpha$ and $E_{a \_R E S E T} / \alpha$, respectively. These results demonstrate that both terms, $E_{a_{-} S E T} / \alpha$ and $E_{a \_ \text {RESET }} / \alpha$, in Eqs. (3) and (4) for the top electrodes follow the order of $\mathrm{Cr}>\mathrm{Au}>\mathrm{Pt}$, indicating that $\mathrm{Pt}$ has the lowest activation energy, $E_{a_{-} S E T}$ and $E_{a_{-} R E S E T}$, for the desorption and adsorption processes, respectively. To put it simply, the chemisorption process is more pronounced if the activation energy of the adsorption/desorption process is reduced, which increases the probability of the generation/elimination of oxygen vacancies. Thus, the $\mathrm{ZnO}$ ReRAM device made with Pt electrodes has a better performance.

In summary, we have demonstrated how surface effects can influence the electrode-dependent resistive switching behavior of $\mathrm{ZnO}$ ReRAM. Both thickness- and ambientdependent electrical properties for each electrode metal studied show that surface effects play an important role on the electrode dependence of the ReRAM performance. The $\mathrm{ZnO}$ ReRAM with Pt electrodes had the lowest activation energy for the oxygen chemisorption process, which allows it to have better switching functionality due to the increased generation/elimination probability of oxygen vacancies. These findings not only provide further insights into the electrode effect on memory switching behaviors but also provide guidance in developing stable ReRAM.

${ }^{1}$ C. Y. Chen, J. R. D. Retamal, I. W. Wu, D. H. Lien, M. W. Chen, Y. Ding, Y. L. Chueh, C. I. Wu, and J. H. He, ACS Nano 6, 9366 (2012).

${ }^{2}$ T. H. Huang, P. K. Yang, W. Y. Chang, J. F. Chien, C. F. Kang, M. J. Chen, and J. H. He, J. Mater. Chem. C 1, 7593 (2013).

${ }^{3}$ W. Y. Chang, C. A. Lin, J. H. He, and T. B. Wu, Appl. Phys. Lett. 96, 242109 (2010).

${ }^{4}$ C. H. Huang, J. S. Huang, S. M. Lin, W. Y. Chang, J. H. He, and Y. L. Chueh, ACS Nano 6, 8407 (2012).

${ }^{5}$ J. W. Seo, J. W. Park, K. S. Lim, J. H. Yang, and S. J. Kang, Appl. Phys. Lett. 93, 223505 (2008).

${ }^{6}$ R. Waser, R. Dittmann, G. Staikov, and K. Szot, Adv. Mater. 21, 2632 (2009).

${ }^{7}$ P. K. Yang, W. Y. Chang, P. Y. Teng, S. F. Jeng, S. J. Lin, P. W. Chiu, and J. H. He, Proc. IEEE 101, 1732 (2013).

${ }^{8}$ J. J. Ke, Z. J. Liu, C. F. Kang, S. J. Lin, and J. H. He, Appl. Phys. Lett. 99, 192106 (2011).

${ }^{9}$ T. H. Huang, P. K. Yang, D. H. Lien, C. F. Kang, M. L. Tsai, Y. L. Chueh, and J. H. He, Sci. Rep. 4, 4402 (2014).

${ }^{10}$ H. Peng, G. Li, J. Ye, Z. Wei, Z. Zhang, D. Wang, G. Xing, and T. Wu, Appl. Phys. Lett. 96, 192113 (2010).

${ }^{11}$ W. H. Xue, W. Xiao, J. Shang, X. X. Chen, X. J. Zhu, L. Pan, H. W. Tan, W. B. Zhang, Z. H. Ji, G. Liu, X. H. Xu, J. Ding, and R. W. Li, Nanotechnology 25, 425204 (2014). 
${ }^{12}$ Y. Han, K. Cho, S. Park, and S. Kim, Trans. Electr. Electron. Mater. 15, 24 (2014)

${ }^{13}$ C. Y. Lin, C. Y. Wu, C. Y. Wu, T. C. Lee, F. L. Yang, C. Hu, and T. Y. Tseng, IEEE Electron Device Lett. 28, 366 (2007).

${ }^{14}$ C. B. Lee, B. S. Kang, A. Benayad, M. J. Lee, S. E. Ahn, K. H. Kim, G. Stefanovich, Y. Park, and I. K. Yoo, Appl. Phys. Lett. 93, 042115 (2008).

${ }^{15}$ Z. J. Liu, J. C. Chou, S. Y. Wei, J. Y. Gan, and T. R. Yew, IEEE Electron Device Lett. 32, 1728 (2011).

${ }^{16}$ W. M. Haynes, CRC Handbook of Chemistry and Physics, 96th ed. (CRC, Boca Raton, FL, 2015-2016), pp. 12-124.

${ }^{17}$ D. H. Lien, J. R. D. Retamal, J. J. Ke, C. F. Kang, and J. H. He, Nanoscale 7, 19874 (2015).

${ }^{18}$ C. Y. Chen, M. W. Chen, J. J. Ke, C. A. Lin, J. R. Retamal, and J. H. He, Pure Appl. Chem. 82, 2055 (2010).

${ }^{19}$ J. J. Ke, K. Namura, J. R. Retamal, C. H. Ho, H. Minamitake, T. C. Wei, D. S. Tsai, C. H. Lin, M. Suzuki, and J. H. He, IEEE Electron Device Lett. 36, 1307 (2015).

${ }^{20}$ M. K. Lee and H. F. Tu, J. Electrochem. Soc. 155, D758 (2008).
${ }^{21}$ L. C. Tien, P. W. Sadik, D. P. Norton, L. F. Voss, S. J. Pearton, H. T. Wang, B. S. Kang, F. Ren, J. Jun, and J. Lin, Appl. Phys. Lett. 87, 222106 (2005).

${ }^{22}$ N. Hongsith, C. Viriyaworasakul, P. Mangkorntong, N. Mangkorntong, and S. Choopun, Ceram. Int. 34, 823 (2008).

${ }^{23}$ B. S. Kang, S. Kim, F. Ren, K. Ip, Y. W. Heo, B. P. Gila, C. R. Abernathy, D. P. Norton, and S. J. Pearton, Appl. Phys. A 80, 259 (2005).

${ }^{24}$ L. Goux, P. Czarnecki, Y. Y. Chen, L. Pantisano, X. P. Wang, R. Degraeve, B. Govoreanu, M. Jurczak, D. Wouters, and L. Altimime, Appl. Phys. Lett. 97, 243509 (2010).

${ }^{25}$ J. K. Jeong, H. W. Yang, J. H. Jeong, Y. G. Mo, and H. D. Kim, Appl. Phys. Lett. 93, 123508 (2008).

${ }^{26}$ S. A. Hoenig and J. R. Lane, Surf. Sci. 11, 163 (1968).

${ }^{27}$ D. Zhang, Mater. Chem. Phys. 45, 248 (1996).

${ }^{28}$ B. Gao, J. F. Kang, H. W. Zhang, B. Sun, B. Chen, L. F. Liu, X. Y. Liu, R. Q. Han, and Y. Y. Wang, in Proceedings of the European Solid-State Device Research Conference (2010), p. 392.

${ }^{29}$ Y. C. Chen, T. C. Chang, H. W. Li, S. C. Chen, J. Lu, W. F. Chung, Y. H. Tai, and T. Y. Tseng, Appl. Phys. Lett. 96, 262104 (2010). 